\section{INTRODUCTION TO PHYSICAL BIOCHEMISTRY}

By J. M. Johlin, Ph.D., D.Sc., Pp. ix +246 . London: Cassell and Co. 1949. 27s. 6d.

This book is more than an introduction to physical biochemistry. It is an elaborate discourse. Starting with the functions and composition of blood there is a useful diagram representing the distribution of the blood constituents playing their role in respiratory exchanges between cells and plasma in arterial and venous blood at rest and at work. A short chapter follows on haemoglobin and chromoproteins. After describing the colligative properties of solutions the author passes to the characteristics of the colloidal state of matter. Here it is of interest to read the names of Graham's nearest contemporaries. We would have liked to read how the osmotic pressure of the blood proteins was measured.
The book in general omits practical details, dealing only with the theoretical aspects. Very useful accounts of chemical equilibrium, the law of mass action, buffer systems, indicators and oxidationreduction potentials are given. There is a good chapter on the respiratory function of the blood followed by acid-base balance. The chapter on oxidation-reduction systems is helped by diagrams of the anaerobic phase of glucose metabolism, the Krebs cycle and cytochrome system.

On entering into details of the more difficult parts the author goes into long calculations which will require careful study by non-mathematical readers, but they are clearly expressed and will be helpful to those passing beyond the elementary descriptions in textbooks of chemistry and physical chemistry for students of medicine and biology.

On the whole the book gives a clear picture of the complicated processes taking part in life, and can be recommended.

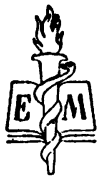

INTERNATIONAL MEDICALABSTRACT SERVICE

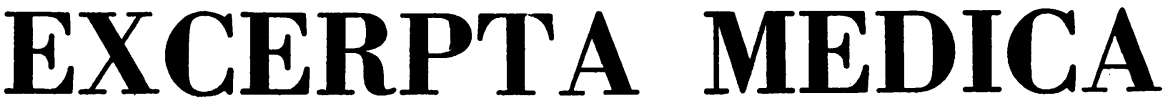

Fifteen separate monthly sectional journals containing abstracts in English from every available medical journal in the world and covering the whole field of clinical and experimental medicine

Write for a prospectus or specimen copy mentioning your speciality E. \& S. LIVINGSTONE, LTD., 16/17 TEVIOT PLACE, EDINBURGH 1

Sole distributors for Great Britain and the British Dominions 